\title{
Guidelines and best practice recommendations on contraception and safe abortion care service provision amid COVID-19 pandemic: Scoping review.
}

\section{Lemi Belay Tolu ( $\square$ lemi.belay@gmail.com )}

1. Saint Paul's Hospital Millennium Medical College, https://orcid.org/0000-0001-6703-828X

Garumma Tolu Feyissa

Jimma University https://orcid.org/0000-0001-6179-0024

Systematic Review

Keywords: COVID-19, Pandemic, Reproductive health, Abortion, Contraception.

Posted Date: April 28th, 2020

DOI: https://doi.org/10.21203/rs.3.rs-25326/v1

License: (1) This work is licensed under a Creative Commons Attribution 4.0 International License. Read Full License 


\section{Abstract}

Introduction: During pandemics there is uncertainty and information overload. Policy makers and health professionals prefer to use summarized evidence of practice recommendations. The aim of this scoping review is therefore to identify available best guidelines, consensus statements, standard of practice and practice recommendations on contraception and safe abortion care service provision during the COVID19 pandemics.

Methods: We searched guideline databases and websites of professional associations and international organizations working on sexual and reproductive health to locate guidelines, protocols, consensus statements and practice recommendations on sexual reproductive health services (SRH) during COVID-19 pandemics. Additionally, we searched: MEDLINE, EMBASE and Google Scholar. We included English records labelled guideline, or recommendation, or consensus, or practice parameter, or position papers on contraception and safe abortion care service practice during the COVID-19 pandemics. Data extraction was done by two independent reviewers using a customized tool that was developed to record the key information of the source that's relevant to the review question. The difference between the two authors on data extraction was resolved by discussion.

Results: Eleven records on safe abortion care and nine records on contraception service were identified. Identified recommendations were categorized into thematic areas. The records addressed approaches to the safe medication and surgical abortion, self-serving family planning and long term and reversible contraception. We haven't employed any of the quality assessment tools as the pandemic is new clinical entity and evidences are based on expert opinion and limited clinical evidence.

Conclusions: Consensus statements and recommendations consistently stated that there should be access to contraception service and safe abortion care during the COVID-19 pandemics with the concerted effort of service re-organization. The practice recommendations focus on innovative ways of service provision to minimize patient and staff exposure to COVID-19 as well as alleviate the burden on the health care system. These include utilizing telemedicine or digital health and community/home-based care or self-care.

\section{Introduction}

The 2019-20 coronavirus pandemic is an ongoing pandemic of coronavirus disease 2019 (COVID-19), caused by severe acute respiratory syndrome coronavirus 2 (SARS-CoV-2)(1). The outbreak was first identified in Wuhan, Hubei Province, China, in December 2019. The World Health Organization (WHO) declared the outbreak to be a Public Health Emergency of International Concern on 30 January 2020 and recognized it as a pandemic on 11 March $2020(2,3)$. 
The WHO considered reproductive health services, including care during pregnancy and child breath as essential health service to continue during the COVID-19 pandemics(4). Additionally, WHO Stated, "Women's choices and rights to sexual and reproductive health care should be respected irrespective of COVID-19 status, including access to contraception and safe abortion to the full extent of the law" (5). But when staff and services are under extreme stress there is a real risk of increasing avoidable harm. The tremendous burden caused by COVID-19 outbreak is exceeding the capacity of many national and local health systems which is jeopardizing routine service delivery and undermining other health priorities. As such the evolving COVID-19 pandemic may affect routine services including sexual, reproductive and maternal health service delivery. Marie Stopes International(MSI) warned near 9.5 million people will miss out reproductive service if service reduction continues for three months because of the lockdown(6). Experience in past epidemics also has shown that lack of access to essential health services and shut down of services unrelated to the epidemic response resulted in more deaths than the epidemic itself(7).

The aim of this scoping review is therefore to identify available guidelines and practice recommendations on provision of safe abortion and contraception service during the COVID-19 pandemics.

\section{Review questions?}

- What's are approaches to maintain safe abortion care service during current COVID-19 pandemics?

- What's are approaches to maintain contraception service during current COVID-19 pandemics?

- What are the available recommendations on service re-organization and staffing of safe abortion and contraception service providers?

\section{Methods And Materials}

\section{Search strategy}

We searched for professional associations and international organizations guidelines, protocols, consensus statements and practice recommendations on safe abortion and contraception services during COVID-19 pandemics. We looked for guideline databases and websites. We searched website of the following associations and organizations: World Health Organization(WHO), America College of Obstetrics and Gynecology (ACOG), Royal College of Obstetrics and Gynecology(RCOG),Royal College of Midwives(RCM), International Federation of Obstetrics and Gynecology(FIGO), Society of Maternal and Fetal Medicine(SMFM), Society of Obstetrics and Gynecology of Canada (SOGC),RANZCOG(The Royal Australian and New Zealand College of Obstetricians and Gynecologists) ,UNICEF (United Nations International Children's Emergency Fund), Faculty of Sexual and Reproductive Healthcare (FSRH), British Society of Abortion Care Providers (BSACP), Society of Family Planning (SFP), United Nations Population Fund(UNFPA), International Planned Parenthood Federation(IPPF) and Marie stopes International(MSI). The guideline data basis searched were: Turning Research into Practice (TRIP) database, Guideline 
International (GIN) library, National Guideline Clearinghouse (NGC) and National Institute for Health and Clinical Excellence (NICE).

In addition to searching the above professional associations and organizations websites and guidelines databases, we also developed search strategy to look any relevant emerging practice recommendations not endorsed by associations and organizations. We searched the following data bases: MEDLINE, EMBASE and Google Scholar (Appendix 1, Search strategy). The report included in this scoping review was prepared based Preferred Reporting Items for Systematic scoping review(8). We considered the following inclusion criteria:

\section{Population.}

This review considered adolescent girls, reproductive age women, women seeking abortion or women or men seeking contraception service, health care providers, health managers and health care institutions.

\section{Interventions.}

The review considered records addressing service delivery approaches and recommendations on safe abortion and contraception service during COVID-19 pandemic.

\section{Context}

The review considered worldwide documents/records addressing safe abortion and contraception service during the COVID-19 pandemic.

\section{Types of documents/records.}

We included records labelled guideline, or recommendation, or consensus, or practice parameter, or position papers on contraception and safe abortion care service practice during the COVID-19 pandemics. The search is limited to English and one year (considering the duration of the outbreak to be after December 2019).

\section{Assessment of methodological quality.}

We haven't employed any of the quality assessment tools (e.g. AGREE II) for clinical practice guidelines as the COVID-19 infection is a relatively new clinical entity where evidences are in the process of emerging and most of the guidelines are based on expert consensus and limited clinical evidences without rigorous guideline development or recommendation synthesis pathways.

\section{Data extraction.}

Data extraction was done by two independent persons using a customized tool that was developed to record the key information of the source that's relevant to the review question. The data extraction tool was developed for guideline related documents, consensus statements and practice recommendations. 
Types of document, timing of publication and summary of recommendations were extracted. The difference between the two authors on data extraction was resolved by discussion. We looked for service delivery organization changes, new position statements and guidelines on safe abortion and contraception services areas in relation to COVID-19 pandemic. We categorized identified guidelines or practice recommendations according to service delivery thematic areas and the findings were described narratively.

\section{Results}

We reviewed 24 websites, four guideline databases, PubMed, EMBASE and Google Scholar. Search yielded a total of 400 records. After removing duplicates, 380 documents were retained for further examination. After screening the titles and abstracts, 36 papers were retained for full-text review. Based on pre-defined inclusion criteria, 15 records were included in the scoping review.

\section{Characteristics of included guidelines and practice recommendations.}

Identified recommendations are categorized into service thematic safe abortion and contraception areas and then into subthemes as first and second trimester, medical or surgical abortion and different contraception types (Table 2).

\section{Key findings.}

- Several international associations and organizations had declared contraception and safe abortion care as essential health service to continue during the COVID-19 pandemics [WHO, ACOG, AMA RCOG, FIGO, RCM, SOGC, RANZCOG, SFP, NAF, IPPF, UNFPA, MSI, BSACP and FSRH].

- Pre-triage (screening) of all clients for Covid-19 is recommended.

- Telemedicine and self-care family planning methods are recommended consistently.

- For women already on combined hormonal contraception (CHC) and progesterone only pills (POP) its recommended to continue 6-12 months without rechecking body mass index (BMI) and blood pressure during the pandemics.

- For long term contraceptive users its recommended to use options of extended use to avoid face to face contact during the pandemics.

- Non-touch or non-test early medication abortion is recommended consistently.

\section{Discussion}

In this review, we attempted to locate documents in the form of guidelines, consensus statements, best practice statements and standards of practice indicating directions on how contraception and safe abortion care service during COVID-19 pandemics. We searched guideline databases, PubMed, EMBASE and Google Scholar and website of international professional associations and organizations working on sexual and reproductive health. 


\section{Contraception service.}

\section{For women already on contraception:}

Telemedicine and self-care family planning methods were recommended consistently. Self-care family planning methods include contraceptive pills, self-injectables, subcutaneous depo shot, condoms, vaginal rings, and fertility awareness methods [WHO, FIGO, RCOG, RCM, SOGC, RANZOG, IPPF, UNFPA, MSI and FSRH ] (3, 9-13).

There are consistent position statements that recommend combined hormonal contraception $(\mathrm{CHC})$ and progesterone only pills (POP) users to continue 6-12 months without rechecking body mass index (BMI) and blood pressure. Depot medroxyprogesterone acetate (DMPA) users can switch to available progesterone only pills (POP) to avoid face to face contact(3, 9, 14, 15). For long term contraceptive user's options of extended use to avoid face to face contact is recommended. Limited evidences show that duration of long acting contraceptive effect is 2 years beyond Food and Drug Administration(FDA)approved duration(16). Depending on that evidences many associations and organizations practice recommendations [FIGO, RCOG, RCM, SOGC, RANZOG, IPPF, UNFPA, MSI and FSRH] advised delaying removal of implants and IUCD during the pandemic crisis unless series side effect happens or wants to get pregnant $(3,9,12,15,17,18)$.

\section{New contraception starters:}

Telemedicine and self-care family planning with remote assessment and prescription of CHC, POP for 612 month and self-injectable contraception were consistently recommended. However, administration of DMPA or insertion of implants or intrauterine device to be considered where concerns about adherence, individual intolerance of oral contraceptives or use of teratogens make longer-acting reversible contraception the only suitable option. Pre-procedure assessment and information-giving remotely to minimize face-to-face contact time (minimum contact service) with healthcare professionals were recommended [WHO, FIGO, RCOG, RCM, SOGC, RANZOG, IPPF, UNFPA, MSI and FSRH]. Optimal use of contact points, such as expanding post-partum family planning with especial focus on long acting reversible contraception were recommended [FIGO, RCOG, RCM, FSRH, MSI and UNFPA].

\section{Emergency contraception (EC):}

Remote assessment of requirement and choice of EC. Oral emergency contraception remote prescription or provision without prescription or Cu-IUD provision with minimum face to face contact is recommended [RCOG, RCM, FSRH, BSACP, FIGO].

\section{Safe abortion service.}

All records (practice recommendations and position papers or commentaries) consistently recommend screening for COVID-19 symptoms from remote before face to face contact or during remote early medication abortion without face to face contact. There were several recommendations on no-touch/no- 
test early medication abortion protocol $(2,3,14,17,19)$. The no-touch protocol depicts pathways to minimize COVID-19 exposure to patient and staffs by organizing early medical abortion services to be delivered via video or teleconferencing /telemedicine and delivery of a treatment package $(2,14,17)$. The treatment package includes mifepristone, misoprostol, ibuprofen and self-care family planning if patient accepted post-abortion contraception. The no-touch/no-test protocol is self-medication abortion in early pregnancy without pre-procedure ultrasound and blood testing. The guideline also indicated that for women in self-isolation because of exposure to COVID-19 no-touch early medication abortion can be arranged similarly at home. If face to face contact care is must for COVID-19 exposed women, the guideline recommends that it should be booked when the isolation period is over unless the gestation is uncertain, and the delay may result in a woman not being able to access abortion in which face to face contact must be arranged with full personal protective measures(14). There is no specific protocol recommended for second trimester medication abortion (above 12 weeks), but professional association and organizations position papers consistently recommend the utilization of telemedicine for digital patient education and counselling to reduce waiting periods and extent of face to face contact(minimal contact service $)(4,17,19,20)$.

For surgical abortion position papers and practice recommendations focus on minimum contact procedure by remote digital patient education, counselling and evaluation. The other focus practice recommendation is increasing safety during the procedure by limiting number of people in the procedure room, appropriate use of personal protective equipment's and decontaminate area after the procedure as per the recommendation (Appendix, see included records). The practice recommendations also include surgical facemask and sanitizer or hand washing for women. Vacuum aspiration, dilatation and evacuation or dilatation and curettage are not aerosol generating procedure unless done by general anesthesia(21). Therefore, these procedures don't require full personal protective equipment's like N95, but abortion provides should screen all patients before the procedure and use standard precautions. Where possible and feasible it's also highlighted to perform the procedures under local anesthesia or intravenous sedation or spinal anesthesia to avoid the need for general anesthesia $(14,17,19,21)$. Its recommended consistently that follow up visits is not required in all conditions, and where needed to be done remotely by telemedicine.

\section{Conclusions}

\section{Implications for practice.}

There were consistent consensus statements and recommendations that there should be access to contraception service and safe abortion care during the COVID-19 pandemics. The practice recommendations focus on minimizing patient and staff exposure to COVID-19 by utilizing telemedicine or digital health and includes the following:

a) No touch/no-taste early medication abortion and minimum contact second trimester medication abortion. b) Minimum contact surgical abortion c) Self-serving contraception methods. d) Minimum 
contact long term contraceptive service provision e) Extended use of long term contraceptive methods.

\section{Implications for research.}

Most of the documents that are included in this review didn't pass through rigorous guideline development process because of the nature of the pandemics. New evidences are evolving with time as duration of the pandemic extends. Hence, we recommend primary studies and systematic reviews to generate evidences on impact of new practices, map and document best practice implementations.

\section{Declarations}

\section{Acknowledgements.}

Not applicable.

\section{Funding.}

This scoping review project did not get any funding support from any organization.

\section{Conflicts of interest.}

The authors declare no conflict of interest in this review.

\section{Authors' contribution.}

Conceptualization: Lemi Belay Tolu, Garumma Tolu Feyissa

Data curation: Lemi Belay Tolu, Garumma Tolu Feyissa

Formal analysis: Lemi Belay Tolu, Garumma Tolu Feyissa

Funding acquisition: NA.

Investigation: Lemi Belay Tolu, Garumma Tolu Feyissa

Methodology: Lemi Belay Tolu, Garumma Tolu Feyissa

Project administration: Lemi Belay Tolu, Garumma Tolu Feyissa

Resources: Lemi Belay Tolu, Garumma Tolu Feyissa

Software: Lemi Belay Tolu, Garumma Tolu Feyissa

Supervision: Lemi Belay Tolu, Garumma Tolu Feyissa

Validation: Lemi Belay Tolu, Garumma Tolu Feyissa 


\section{References}

1. Adhikari SP, Meng S, Wu Y-J, Mao Y-P, Ye R-X, Wang Q-Z, et al. Epidemiology, causes, clinical manifestation and diagnosis, prevention and control of coronavirus disease (COVID-19) during the early outbreak period: a scoping review. Infectious Diseases of Poverty. 2020;9(1):1-12.

2. No Touch Medication Abortion Protocol [Available from: https://www.reproductiveaccess.org/resource/no-touch-mab-protocol/.

3. RANZCOG - COVID-19: Access to reproductive health services [Available from: https://ranzcog.edu.au/news/covid-19-access-to-reproductive-health-services.

4. World Health Organization. COVID-19: operational guidance for maintaining essential health services during an outbreak: interim guidance, 25 March 2020. World Health Organization.

5. World Health Organization. Clinical management of severe acute respiratory infection (SARI) when COVID-19 disease is suspected: interim guidance, 13 March 2020. World Health Organization.

6. Marie Stopes International (MSI) response to the COVID-19 crisis \%U https://www.mariestopes.org/covid-19.

7. Sochas L, Channon AA, Nam S. Counting indirect crisis-related deaths in the context of a lowresilience health system: the case of maternal and neonatal health during the Ebola epidemic in Sierra Leone. Health policy and planning. 2017;32(suppl_3):iii32-iii9.

8. Tricco AC LE, Zarin W, O'Brien KK, Colquhoun H, Levac D, et al. PRISMA Extension for Scoping Reviews (PRISMAScR): Checklist and Explanation. Ann Intern Med.169:467-73.

9. Reproductive acces project. Contraception in the Time of COVID-19 [Available from: https://www.reproductiveaccess.org/resource/contraception-covid/.

10. FSRH CEU: information to support management of individuals requesting to discontinue contraception to plan a pregnancy during the Covid-19 outbreak (26 March 2020) - Faculty of Sexual and Reproductive Healthcare [Available from: https://www.fsrh.org/documents/fsrh-ceu-informationto-support-management-of-individuals/.

11. World Health Organization (WHO). Contraception/ family planning, and COVID-19\%U https://www.who.int/news-room/q-a-detail/contraception-family-planning-and-covid-19.

12. FIGO. COVID-19 Contraception and Family Planning. https://www.figo.org/covid-19-contraceptionand-family-planning [Available from: https://www.rcog.org.uk/en/guidelines-researchservices/guidelines/coronavirus-pregnancy/covid-19-virus-infection-and-pregnancy/.

13. Sexual and Reproductive Health and Rights: Modern Contraceptives and Other Medical Supply Needs, Including for COVID-19 Prevention, Protection and Response [Available from: /resources/sexual-and-reproductive-health-and-rights-modern-contraceptives-and-other-medicalsupply.

14. Royal College of Obstetricians and Gynaecologists, Faculty of Sexual and Reproductive Healthcare and the British Society and Abortion Care Providers.Coronavirus (COVID-19) infection and abortion 
care \%U https://www.rcog.org.uk/en/guidelines-research-services/guidelines/coronavirus-abortion. Royal College of Obstetricians \&amp; Gynaecologists.

15. Faculty of Sexual \& Reproductive Healthcare. FSRH CEU clinical advice to support provision of effective contraception during COVID-19 outbreak. 20 March 2020. Available: https://www.fsrh.org/documents/fsrh-ceu-clinical-advice-to-support-provision-of-effective/

16. McNicholas C, Swor E, Wan L, Peipert JF. Prolonged use of the etonogestrel implant and levonorgestrel intrauterine device: 2 years beyond Food and Drug Administration-approved duration. American journal of obstetrics and gynecology. 2017;216(6):586.e1-.e6.

17. FSRH, RCOG, RCM and BSACP launch joint guidance on COVID-19 and abortion care - Faculty of Sexual and Reproductive Healthcare \%U https://www.fsrh.org/news/fsrh-rcog-and-bsacp-guidanceon-covid-19-abortion.

18. FSRH, RCOG \& RCM statement provision of postpartum contraception during Covid-19 - Faculty of Sexual and Reproductive Healthcare [Available from: https://www.fsrh.org/documents/fsrh-rcogrcm-statement-postpartum-contraception-covid19/.

19. National Abortion Federation. Abortion \& COVID-19 [Available from: https://prochoice.org/abortioncovid-19/.

20. FSRH Position: Essential SRH Services during COVID-19 March 2020 - Faculty of Sexual and Reproductive Healthcare \%U https://www.fsrh.org/documents/fsrh-position-essential-srh-servicesduring-covid-19-march-2020.

21. American College of Surgeons. COVID-19: Elective Case Triage Guidelines for Surgical Care,March 24,2020 [Available from: https://www.facs.org/covid-19/clinical-guidance/elective-case.

\section{Appendix 1: Tables}

Table 1: Search strategy; Search conducted on April 18,2020, MEDLINE (Ovid).

\begin{tabular}{|l|l|l|}
\hline Number & Search Query & Result. \\
\hline 1 & COVID-19 [tw] OR Sars cov-2[tw] OR Pandemics[tw] OR epidemics [tw] AND maternal care[tw] OR reproductive & 92,0931 \\
& service[tw] OR abortion [tw] OR contraception[tw] OR family planning[tw] & \\
\hline 2 & Limit 1 to English AND Human AND 1 year. & 400 \\
\hline
\end{tabular}

Table 2: Characteristics of identified records and common practice recommendations. 


\begin{tabular}{|c|c|c|c|c|}
\hline \multicolumn{2}{|c|}{ Practice area and common recommendations } & \multicolumn{2}{|r|}{ Sources and name of the record } & \multirow{2}{*}{$\begin{array}{l}\text { Types of document (guideline, } \\
\text { commentaires, position statements) } \\
\text { Guidance, position statements and } \\
\text { commentaries. }\end{array}$} \\
\hline Safe abortion & $\begin{array}{l}\text { No-touch / no-test early } \\
\text { medication abortion. }\end{array}$ & 11 & $\begin{array}{l}\text { FIGO, RCM, SOGC, RCOG, } \\
\text { RANZCOG, } \\
\text { NAF, IPPF, UNFPA, MSI, FSRH, } \\
\text { BSACP }\end{array}$ & \\
\hline & $\begin{array}{l}\text { Minimum contact second } \\
\text { trimester medication abortion. }\end{array}$ & 11 & $\begin{array}{l}\text { FIGO, RCM, SOGC, RCOG, } \\
\text { RANZCOG, } \\
\text { NAF, IPPF, UNFPA, MSI, FSRH, } \\
\text { BSACP }\end{array}$ & $\begin{array}{l}\text { Guidance, position statements and } \\
\text { commentaries }\end{array}$ \\
\hline & $\begin{array}{l}\text { Minimum contact surgical } \\
\text { abortion. }\end{array}$ & 11 & $\begin{array}{l}\text { FIGO, RCM, SOGC, RCOG, } \\
\text { RANZCOG, } \\
\text { NAF, IPPF, UNFPA, MSI, FSRH, } \\
\text { BSACP }\end{array}$ & $\begin{array}{l}\text { Guidance, position statements and } \\
\text { commentaries }\end{array}$ \\
\hline \multirow[t]{3}{*}{ Contraception } & $\begin{array}{l}\text { Self-serving contraception } \\
\text { methods. }\end{array}$ & 9 & $\begin{array}{l}\text { FIGO, WHO, RCM, RCOG, FSRH, } \\
\text { IPPF, UNFPA, MSI, RANZCOG }\end{array}$ & $\begin{array}{l}\text { Guidance, position statements and } \\
\text { commentaries }\end{array}$ \\
\hline & $\begin{array}{l}\text { Extended use of long term } \\
\text { contraceptives }\end{array}$ & 7 & $\begin{array}{l}\text { RCM, RCOG, FSRH, IPPF, } \\
\text { UNFPA, MSI, RANZCOG }\end{array}$ & $\begin{array}{l}\text { Guidance, position statements and } \\
\text { commentaries }\end{array}$ \\
\hline & $\begin{array}{l}\text { Minimum contact long term } \\
\text { contraceptives. }\end{array}$ & 8 & $\begin{array}{l}\text { FIGO, RCM, RCOG, FSRH, IPPF, } \\
\text { UNFPA, MSI, RANZCOG }\end{array}$ & $\begin{array}{l}\text { Guidance, position statements and } \\
\text { commentaries }\end{array}$ \\
\hline
\end{tabular}

\section{Appendix 2: Supplementary Materials}

1. S2 document: Data extraction tool (safe abortion and contraception service).

2. S2 Table: Scoping review PRISMA checklist. It describes the review against the checklist for PRISMA reporting guideline.

\section{Figures}




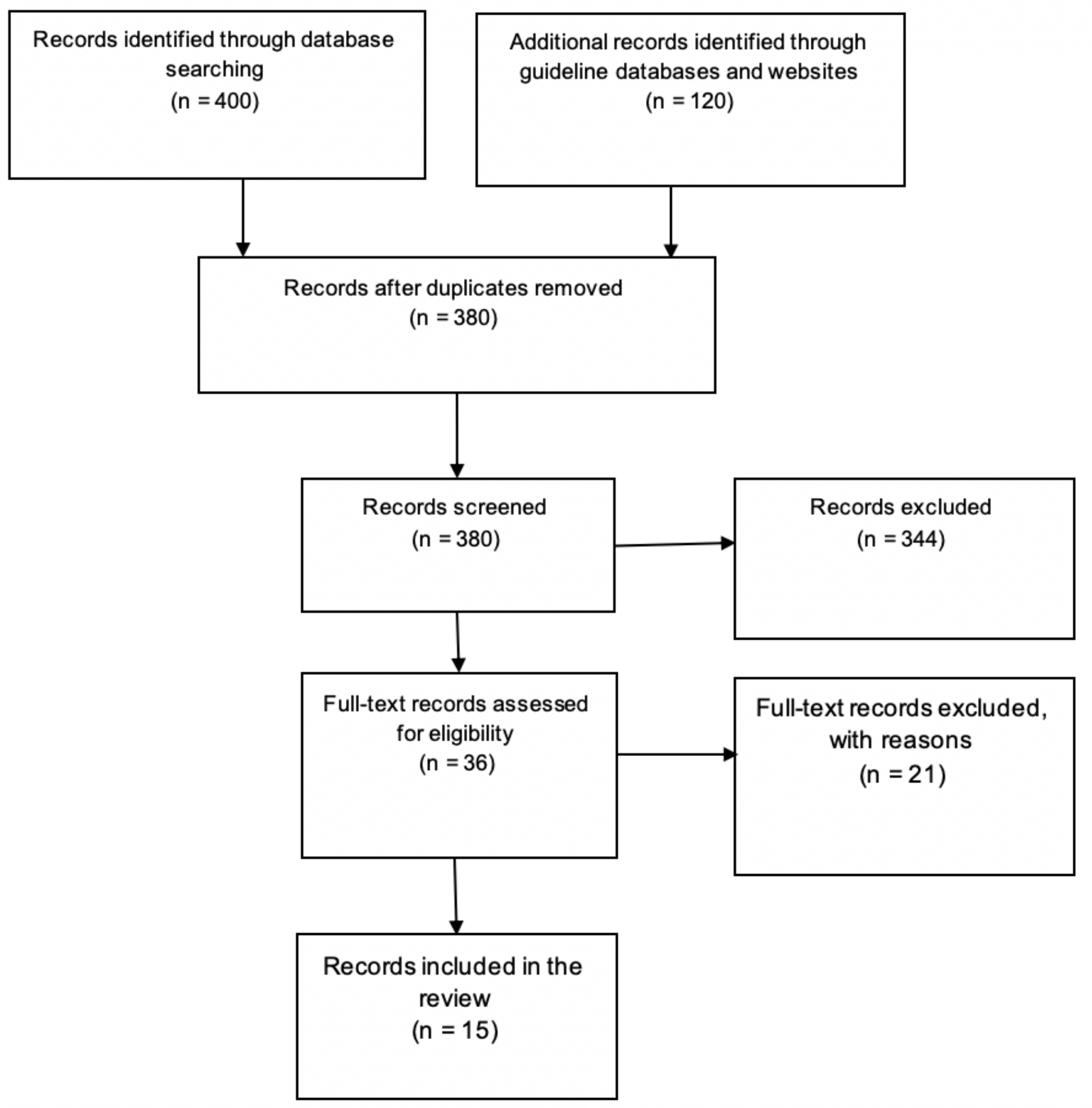

Figure 1

Study selection process. From: Moher D, Liberati A, Tetzlaff J, Altman DG, The PRISMA Group (2009) Preferred Reporting Items for Systematic Reviews and Meta-Analyses: The PRISMA Statement. PLoS Med 6(7): e1000097. https://doi.org/10.1371/journal.pmed.1000097.

\section{Supplementary Files}


This is a list of supplementary files associated with this preprint. Click to download.

- S1ExcelDocumentDataextractiontoolsafeabortioncontraceptionservice..xlsx

- S1tablePRISMAScR.docx 